\title{
E1 valor de habla peculiar en la lingüística de hoy y de (casi) siempre
}

\author{
Milagros Fernández PÉRez \\ Universidade de Santiago de Compostela \\ Facultad de Filología \\ Campus Norte - Burgo das Nacións \\ 15782 Santiago de Compostela (Galicia) \\ E-mail: magos.fernandez.perez@usc.es
}

\section{EL VALOR DE HABLA PECULIAR EN LA LINGÜISTICA DE HOY Y DE (CASI) SIEMPRE}

RESUMEN: El habla peculiar tiene su lugar reconocido en las coordenadas de la llamada Lingüística de los usos. Las producciones verbales, sean o no normativas, adquieren relieve y deben examinarse. Se trate de verbalizaciones infantiles en etapa de desarrollo, se trate de producciones especiales por déficit, sean producciones por limitación en destreza, en cualquiera de los casos, funcionan patrones sistemáticos que dan soporte a códigos propios y que deben ser atendidos en linguiistica. Es objetivo de este trabajo mostrar los derroteros por los que ha circulado la atribución de interés intrínseco al habla peculiar en la linguilstica de ayer y con atención especial a la linguística más reciente. Se subrayan así factores prácticos como detonantes de la necesidad de análisis privativos del habla singular (en §2), y se destacan aportaciones de enfoque (en §3.1) y de teoría y método (en §3.2) que dan carta de naturaleza al habla peculiar por su valor exclusivo. A modo de corolario se recogen las conclusiones de la contribución en $\$ 4$.

PALABRAS CLAVES: habla peculiar; desarrollo de la lengua; patologías del lenguaje; lingüística de los usos.

SUMARIO: 1. Introducción. la lingüística más reciente en contexto y la parole como objeto de estudio. 2. ¿Qué mueve el interés por el habla peculiar? su valor intrínseco y algunas esferas de relevancia. 3. El habla peculiar en la lingüistica de hoy. Interés descriptivo y teórico. 4. Conclusiones. A modo de corolario.

\author{
THE VALUE OF PECULIAR \\ SPEECH IN THE LINGUISTICS \\ OF TODAY AND (ALMOST) \\ ALWAYS
}

ABSTRACT: Peculiar speech has claimed its own place within the socalled Usage-based Linguistics. Verbal utterances, standard or otherwise, acquire importance and should be examined. $\mathrm{Be}$ it child utterances in their developmental stages; be it special, impaired roductions; be it productions with a limited ability; in any case, sistematic patterns are at work which support their own codes and should be studied in Linguistics. It is the goal of this paper to show the paths by which intrinsic interest to peculiar speech has been given by Linguistics in the past and in the most recent days. We highlight practical aspects as catalysts of the necessity of analysis of peculiar speech (in \$2), and we outline contributions related to the approach (§3.1) and to theory and method (in §3.2) as elements which acknowledge the exclusive value of peculia speech. As a corollary, conclusions of this work are presented in $\$ 4$.

KEY WORDS: atypical speech; language development; language pathology; usage-based linguistics.

SUMMARY: 1. Introduction. The most recent Linguistics and the parole as a subject of study. 2.What motivates the interest for peculiar speech? Its intrinsic value and some areas of interest. 3. Peculiar speech in the Linguistics of today. Descriptive and theoretical interest. 3.1. Some milestones in the assessment of peculiar speech. 3.2. Theoretical approaches in Linguistics and the relevance of peculiar speech. 4. Conclusions. Corollary.

\section{LA RELEVANCE DU LANGAGE PARTICULIER DANS LA LINGUISTIQUE D'AUJOURD'HUI ET DE (PRESQUE) TOUJOURS}

RÉSUMÉ : La parole particulier a son lieu propre dans le contexte de la dite Linguistique des usages. Les productions verbales, réglementaires ou pas réglementaires, sont mises en lumière et doivent être examinées. Sois verbalisations des enfants à l'étage de développement, sois productions spéciaux par dysfonction, sois paroles résultantes d'une compétence faible, toujours, dans tous les cas, il y a des modèles qui soutiennent des codes propres qui doivent être examinés en Linguistique. Dans ce travail on montre les parcourus de l'attribution d'intérêt intrinsèque à la parole particulier dans la linguistique d'hier et surtout avec spécial attention à la linguistique d'aujourd'hui. On souligne, comme ça, des éléments pragmatiques comme déclencheurs de l'analyse privative du language singulier (§2), et aussi on note des approches (§3.1) et des contributions théoriques et methodologiques (§3.2) qui ont participé à la fondation de la rélévance du langage particulier. Il y a finalement un corollaire qui contient les conclusions du travail en $\S 4$

MOTS CLÉS: parole atypique développment du langage; pathologie linguistique; linguistique des usages.

SOMMAIRE: 1.Introduction. La Linguistique d'aujourd'hui et la parole comme objet d'étude. 2. Pourquoi l'intérêt par la parole particulier? Sa valeur intrinsèque et certains domains de rélévance. 3 . La parole spécial dans la Linguistique actuelle. Son intérêt descriptif et théorique. 3.1. Quelques jalons à la appréciation de la parole particulier. 3.2. Approches théoriques en Linguistique et rélévance de la parole spécial. 4. Conclusion. À titre de corollaire
Fecha de Recepción Fecha de Revisión Fecha de Aceptación Fecha de Publicación
$05 / 02 / 2019$

$22 / 02 / 2019$

$10 / 05 / 2019$

$01 / 12 / 2020$ 


\title{
E1 valor de habla peculiar en la lingüistica de hoy y de (casi) siempre ${ }^{1}$
}

\author{
Milagros FernÁNDEZ PÉREZ
}

\section{INTRODUCCIÓN. LA LINGÜÍSTICA MÁS RECIENTE EN CONTEXTO Y LA PAROLE COMO OBJETO DE ESTUDIO}

El habla singular que no se acomoda al patrón normativo esperable no es común que se haya contemplado en estudios superiores de filología o de lingüística. Lo que habitualmente se toma como objeto para enseñar y para describir es el modelo estándar basado en el molde de lengua escrita. Sea la langue saussureana, o el sistema coseriano en los planteamientos estructuralistas, sea la competencia del hablante-oyente ideal en concepciones generativistas. Ahora bien, que no se haya abordado el habla peculiar no quiere decir que no tenga interés. Quizás no se hayan dado las condiciones indispensables para que las formas de expresarse y los usos especiales para hacerse entender, se consideren en su relevancia y se estudien en sus detalles. Sin duda, ha tenido que producirse un giro notable en el modo de hacer lingüistica, con el cambio relativamente brusco de la lingüística monolítica del sistema hacia una lingüística del habla, ocupada de los usos y centrada en las variedades antes que en la norma prevista. Ese viraje algo vertiginoso en el campo de las ciencias del lenguaje hay que contextualizarlo en coordenadas de avances técnicos y cambios sociales que reorientan los intereses y las posibilidades de investigación. Más concretamente, son las nuevas tecnologías y los desarrollos informáticos los que permiten dibujar una nueva lingüística de campo traducida en registros de muestras verbales y en acopio de datos en grandes repertorios. En términos de sociología de la ciencia, la llamada lingüística de corpus va de la mano de objetivos aplicados con el punto de mira puesto en la utilidad y en la transferencia de resultados. Las variedades sociolingüisticas visibles en la investigación lingüística desde la segunda mitad de la década de los años $70 \mathrm{y}$ las reglas pragmático-comunicativas focalizadas en su pertinencia en las prácticas verbales desde finales de los años 80, se convierten en verdaderas protagonistas con el desarrollo de la lingüistica de corpus. Es su cultivo el que facilita el acopio de materiales y su tratamiento y gestión. Los usos lingüísticos se han convertido en los protagonistas estrella de la investigación lingüística. De algún modo se difumina y se borra la lingüística in vitro y germina la lingüística in vivo.

Es en el marco de estas coordenadas de la lingüística más reciente en donde el habla peculiar tiene su lugar. Las producciones verbales, sean o no

\footnotetext{
${ }^{1}$ Este trabajo se inscribe en el marco del proyecto de investigación Adquisición fónica y corpus. Tratamiento en PHON del corpus koiné de habla infantil (FFFI2017-82752-P), financiado por FEDER / Ministerio de Ciencia, Innovación y Universidades - Agencia Estatal de Investigación, en la convocatoria de Excelencia de 2017.
} 
normativas, adquieren relieve y deben examinarse. Se trate de producciones infantiles en etapa de desarrollo, se trate de producciones especiales por déficit o retroceso, o sean producciones por limitación en destreza, en cualquiera de los casos, existen patrones sistemáticos que dan soporte a códigos propios. Y esto ciertamente debe ser atendido en lingüística.

En su libro de 1980, Introduction to Language Pathology, Crystal afirmaba con claridad meridiana que la tarea del lingüista en la esfera de las disfunciones comunicativas es precisar el sistema de reglas que manejan los sujetos afectados:

No existe modo de saber por adelantado si el sistema que vamos a encontrar es un reflejo del sistema presente en la comunidad a la que el paciente pertenece (...). La tarea del patólogo del habla es determinar cuáles son esas reglas (...). Es un poco como estar haciendo un rompecabezas que puede encajar de muchos modos diferentes para dar lugar a distintos modelos (Crystal, 1980: 63).

De modo similar lo defiende también Givón en un destacado trabajo de 2002 sobre el procesamiento visual como precursor evolutivo del lenguaje, en donde el autor afirma la posibilidad humana de comunicarse aun sin gramática plena y tan solo con léxico y combinatoria (cuadro 1).

\begin{tabular}{|c|c|c|}
\hline PROPIEDADES & DISCURSO Y GRAMÁTICA & DISCURSO Y PRE-GRAMÁTICA \\
\hline $\begin{array}{l}\text { Estructurales } \\
\text { a. Morfología gramatical } \\
\text { b. Construcciones sintácticas } \\
\text { c. Orden de palabra } \\
\text { d. Pausas }\end{array}$ & $\begin{array}{l}\text { Abundante } \\
\text { Complejas/Incrustadas } \\
\text { Gramatical } \\
\text { Fluente }\end{array}$ & $\begin{array}{l}\text { Ausente } \\
\text { Simples/yuxtapuestas } \\
\text { Pragmático } \\
\text { Vacilante }\end{array}$ \\
\hline $\begin{array}{l}\text { Funcionales } \\
\text { e. Velocidad procesamiento } \\
\text { f. Esfuerzo mental } \\
\text { g. Dependencia contexto }\end{array}$ & Rápido Bajo Bajo & Lento Alto Alto \\
\hline $\begin{array}{l}\text { Cognitivas } \\
\text { h. Modo procesamiento } \\
\text { i. Adquisición } \\
\text { j. Evolución }\end{array}$ & $\begin{array}{l}\text { Automatizado } \\
\text { Tardia } \\
\text { Tardia }\end{array}$ & Asistido Temprana Temprana \\
\hline
\end{tabular}

CUADRO 1: Procesamiento del discurso en gramática de lengua-producto y en pre-gramática de lengua emergente (adaptado de Givón, 2002: 11)

La proto-gramática, no obstante, contiene reglas (de entonación, de espaciado y distancia, de secuenciación y orden y de cantidad, Givón, 2002: 14-15) que "they perform exactly the same functions in grammaticalized language as they do in pre-grammaticalized communication. Nothing has been lost, but rather some things have been added" (Givón, 2002: 15). 
El objetivo de nuestro trabajo es mostrar los derroteros por los que ha circulado la atribución de interés intrínseco al habla peculiar en la lingüística de ayer y con atención especial a la lingüística más reciente. Se subrayan algunos factores prácticos como detonantes de la necesidad de análisis privativos del habla singular (en el §2), y se destacan ciertas aportaciones de enfoque (en el §3.1) y de teoría y método (en el §3.2) que dan carta de naturaleza al habla peculiar por su valor exclusivo. Se recogen a modo de corolario las principales conclusiones de la contribución en el §4.

\section{2. ¿QUÉ MUEVE EL INTERÉS POR EL HABLA PECULIAR? SU VALOR INTRÍNSECO Y ALGUNAS ESFERAS DE RELEVANCIA}

Si rastreamos a través de la historia de la lingüística, las motivaciones de atención al habla atípica han estado sobre todo en tratar de solventar las limitaciones o los problemas de comunicación que se desencadenan. Esto explica que ciertas dimensiones que hacen patente la dificultad para hacerse entender, como la pronunciación, la fluidez o la ausencia de producción oral, hayan sido consideradas desde siglos atrás. Expertos reconocidos como Bonet (1573-1633, Reduction de las letras $y$ arte para enseñar a hablar a los mudos, 1620) y Bulwer (1606-1656, Chirologia, or The Natural Language of the Hand, 1644) elaboraron métodos para hablar con sordos. Asimismo, Rousselot (1846-1924) y Grammont (1866-1946) se ocuparon de defectos de pronunciación y diseñaron instrumentos de observación que contienen el germen de la fonética moderna. Como no podia ser de otro modo, los inicios institucionales de los llamados trastornos comunicativos se aposentan en el campo de la Terapéutica del habla. Las primeras actividades y asociaciones de especialistas ocupados en temas de expresión oral peculiar que debe repararse se organizan en Berlín, en donde en 1886 se crea la primera Escuela de Terapia del habla y de la voz y en 1891 surge la primera Asociación de profesionales en Terapia del habla (cfr. Eldridge, 1968) ${ }^{2}$. En 1924 se celebra en Viena el primer encuentro de la Asociación Internacional de Logopedia y Foniatría y en 1925 se instaura en Estados Unidos la Academia para la corrección del habla, y a partir de 1936 se inicia la andadura editorial de los órganos más representativos del ámbito: Journal of Speech Disorders; Journal of Speech and Hearing Disorders (en la actualidad, Journal of Speech and Hearing Research).

En la lingüística británica la importancia del habla peculiar y su orientación hacia la Terapia del habla corre paralela al origen, crecimiento y arraigo sólido del trabajo en The London School of Phonetics. Los trabajos de Templin (1957) y de Morley (1957) sobre dislalias y destrezas verbales en niños resultan significativos de esta tendencia. Se propone la distinción capital entre delayed speech y disordered speech, que sin duda trasluce la

\footnotetext{
${ }^{2}$ Como señala Eldridge (1968: 5): "Germany and Austria were especially prolific in literature concerning the many varieties of speech and voice disorder before the First World War".
} 
necesidad de atender a las características propias del habla en desarrollo. No todas las pronunciaciones infantiles constituyen "disfunción", sino que puede haber "demoras" y, para dilucidarlo, se hacen imprescindibles datos sobre fases en el progreso adquisitivo. Se ha abierto asi la espita para dar valor en sí misma al habla infantil.

En lo que se refiere a la tradición hispana más cercana, sobresale la aportación de Barnils (1982-1933), fonetista-ortofonista catalán que entronca con la orientación de la terapéutica del habla cuyo objetivo consiste en corregir deficiencias de pronunciación, tal y como señala en su libro Defectes del parlar (1930: 9): "la majoria dels trastorns locutius que hi registrem, poden tractar-se i corregir-se sense appellar a altres recursos que als exercicis adecuats". Pero, sobre todo, y en la linea de reconocer la importancia del lenguaje genuino de los niños como materia de estudio, destacan entre nosotros las figuras de Gili Gaya y de Alarcos Llorach. El discurso de Gili Gaya con motivo de su entrada a la Academia de la Lengua en 1961 versó sobre Imitación y creación en el lenguaje infantil, y en él se contienen afirmaciones como la siguiente:

La palabra infantil no debe ser mirada como simple esbozo de algo más perfecto que vendrá después, sino como un decir estructurado y valioso de por sí (...). El maestro que pretenda dirigir la actividad expresiva de los niños debe aprender primero a conocerla y respetarla. Y esta es la lección que mis discípulos grandes y chicos me enseñaron, y hoy he venido a recitar ante la Academia (Gili Gaya, 1961: 31, resaltado nuestro)

Alarcos Llorach abordó el tema de La adquisición del lenguaje por el niño en un trabajo de 1968 incluido en el compendio 3 del Tratado del lenguaje dirigido por Martinet. Se trata de un estudio general sobre el desarrollo fónico, gramatical y léxico de la lengua en las primeras etapas, si bien hay apreciaciones teóricas interesantes sobre el carácter evolutivo del lenguaje infantil. En su opinión,

El estudio de la lengua infantil es uno de los campos donde más visible resulta la maraña fundamental de los aspectos diacrónico y sincrónico del lenguaje. A primera vista, parece que deberiamos encararlo según los métodos de la evolución propios de la diacronía. Pero si el observador se limita simplemente a efectuar la exposición cronológica de los fenómenos que se producen durante los años de aprendizaje de un idioma, no podremos comprender las líneas generales, el sentido, ni el sistema del proceso. Sin dejar de tener en cuenta la sucesión en el tiempo, es necesario señalar las relaciones que existen entre los hechos simultáneos, es decir, el funcionamiento sincrónico de los elementos constitutivos de la lengua infantil (Alarcos, 1968: 9-10).

Si bien el reconocimiento y la visibilidad institucional del lenguaje peculiar se ha canalizado a través de dislalias y disfunciones -tal y como se constata en la prominente escuela británica-, ello no quiere decir que no existan aproximaciones que se interesen por la vertiente simbólica del lenguaje ${ }^{3}$. El crucial papel de la gramática en la elaboración de mensajes

\footnotetext{
${ }^{3}$ En cualquier caso, la elaboración de un "Alfabeto Fonético para el habla peculiar» (extIPA Symbols for Disordered Speech:
} 
atípicos se ha destacado ya desde los primeros casos de sujetos con lesiones cerebrales, si bien en buena parte de los estudios el enfoque ha sido por "loque-se-ha-perdido", "lo-que-le-falta" respecto del lenguaje esperado. Quizás porque en estas circunstancias el objetivo primordial no es tanto la terapéutica y la recuperación cuanto el estudio teórico y descriptivo del procesamiento cerebral y mental del lenguaje. La afasiología lingüistica prevalece, con sus investigaciones sobre la fiabilidad de los datos y la validez de las inferencias, sobre la clinica y las rutas de tratamiento (Caplan, 1987). Como es natural, primero ha de lograrse el conocimiento teórico y se han de diseñar los modelos apropiados, y solo después se definen vías terapéuticas (Howard y Hatfield, 1987) 4 .

En sintesis, los objetivos, sean aplicados y terapéuticos, sean teóricos y de diseño de modelos de procesamiento, inciden de algún modo en la consideración del habla peculiar. Para hacer terapia parece necesario dar valor a las singularidades que manifiestan los sujetos en sus usos comunicativos y con objeto de aprovechar sus posibilidades. Goldstein (1947: 329), destacado neuropsicólogo, es rotundo cuando dice que "It is much more important for him to be able to use his speech for comprehensible communication than to speak correctly". Sin embargo, para abordar en términos teóricos de procesamiento del lenguaje las situaciones atípicas, el enfoque casi siempre es un modelo 'normal' de la lengua, de modo que el lenguaje singular y sus procesos se contemplan como meras disfunciones. Se producirá un cambio de consideración una vez que se reconozca que el cerebro es un órgano (y no únicamente una masa con áreas localizadas), que puede redefinir funciones y de este modo quizás recuperar las destrezas perdidas. Las contribuciones de Jakobson (1941) y de Luria (1975) son significativas de la estela ya esbozada por Jackson (18795). Donde Jakobson dice que el estudio del lenguaje afásico ha de conducir a una redistribución de las funciones en el cerebro (Jakobson, 1941), Luria (1975: 353) apunta el dinamismo de los procesos y la necesidad de contemplarlos en su interrelación global:

\footnotetext{
The 'centers' of traditional neuropsychology are rather to be considered as levels by means of which cognition is carried one stage further. Similarly, pathways do not serve to associate ideas, perceptions to movements, written words to sounds, etc., but rather link up temporally transformations occurring at different points in the microgenetic sequence. Cognition is a lawful unfolding of evolutionary forms, not a pastiche of more or less fragmentary elements.
}

https://www.internationalphoneticassociation.org/sites/default/files/extIPA_2016.pdf), al abrigo de la International Phonetic Association

(https://www.internationalphoneticassociation.org/), es prueba fehaciente del reconocimiento descriptivo y teórico de la dimensión fónica del habla disfuncional.

${ }^{4}$ Ya en 1947 Zanwigll era contundente a este respecto: "No method of treatment is better than the principles on which it is based, and the search for principles should concern us no less than the immediate clinical situation" (1947: 7).

${ }^{5}$ Cognition is a lawful unfolding of evolutionary forms, not a pastiche of more or less fragmentary elements. 


\section{El HABLA PECULIAR EN LA LINGÜÍSTICA DE HOY. INTERÉS DESCRIPTIVO Y TEÓRICO}

Algunos trabajos que se han convertido en hitos, y asimismo ciertas corrientes en Lingüística, han marcado puntos de inflexión en las rutas para abordar el lenguaje peculiar en su naturaleza propia y para darle valor e importancia en su significación genuina. Se trata en cualquier caso de planteamientos que promueven la relevancia del habla y defienden la riqueza de los datos como fuentes que sustentan la investigación y la teoría. Todos ellos en el marco de la lingüística de corpus y bajo el paraguas de la lingüística cognitiva de usos y construcciones.

\subsection{AlgunOS hitos EN LA VALORACIÓN DEL HABLA PECULIAR}

Entre las contribuciones pioneras sobresale la investigación compendiada en el volumen Agrammatic Aphasia. A Cross-Language Narrrative Sourcebook y editada por Menn y Obler, en la que los usos verbales de afásicos se contemplan en su realidad interidiomática, que es diversa, pero también con dosis de efectividad y alcance comunicativo en cada caso $^{6}$. Se aportan datos de producciones de pacientes afásicos en catorce lenguas tipológicamente variadas (desde el islandés hasta el hindi pasando por el polaco, el hebreo o el finés). Del análisis de las producciones reales deriva la ruptura con el concepto homogéneo de agramatismo al constatar su variación dependiente de la lengua. De ahí que el eje central que ha motivado la pesquisa gire en torno a la cuestión: "in what way do the grammars of particular languages modify the manifestations of this disorder?"7 (Menn y Obler, 1990: 1387).

\footnotetext{
${ }^{6}$ Los estudios de afasias limitados a lenguas genéticamente emparentadas no ofrecen garantías para decidir entre alternativas teóricas. Con palabras de Menn y Obler (1990: 1369), "It is not posible to choose among the alternative theoretical explanations of agrammatic aphasia only in the basis of data from the handful of languages (English, French, German, Italian, Russian) that have been the principal objects of aphasiological study until now. This book was created to help in choosing among and considering the alternatives".

${ }^{7} \mathrm{El}$ procedimiento comparativo en lingüistica se vuelve clave para atribuir garantías a las generalizaciones y para arrojar luz en situaciones problemáticas. Con palabras de Menn y Obler (1990: 8):
}

In all areas of research involving language and its uses, cross-language comparisons play the essential role of making it posible to distinguish valid from spurious generalizations, by permitting the examination of a wide variety of combinations of linguistic devices"-(1990: 8).

Cross-language studies are essential in this regard because data that are ambiguous as to level of impairement in one language may quite clear in another. For example, phonological, morphological, and syntactic accounts of errors in agrammatism are all attractive for English, but as Grodzinsky (1984) has shown, phonological accounts are inadequate for Hebrew"-(1990: 9). 
El propósito metodológico de fundamentar en una base empírica amplia la teoria sobre el agramatismo ha conducido a la necesidad de describir en su realidad el habla de afásicos en distintos idiomas, de modo que el habla peculiar disfuncional cobra importancia en sí misma. Y no únicamente porque se haya de prestar atención descriptiva a las producciones, sino en mayor medida porque los resultados del análisis tienen relevancia crucial en la teoria. De hecho, las evidencias derivadas del trabajo descriptivo han obligado a replantear la noción misma de agramatismo en el sentido de matizarla para dar cabida a las 'gramáticas especiales' que construyen los pacientes afásicos en las distintas lenguas. Con palabras de Menn y Obler (1990: 1371):

The patients who appeared more severely aphasic overall omitted a greater percentage of free grammatical morphemes; patients also showed some substitution errors on these morphemes. However, other features of agrammatism as we know it from betterstudied languages, especially the omission of bound grammatical morphemes and the use of infinitive verb forms, appear to vary in accord with differences in the grammars of the different languages.

Los pacientes afásicos componen gramáticas que se elaboran según las propiedades estructurales de la morfosintaxis particular de la lengua en cada caso. No siempre se omiten morfemas, puede que se sustituyan por otros. No conviene mantener que desaparece el orden de las palabras en las estructuras sintácticas, hay que incluir la posibilidad de que se defina un nuevo orden. Tampoco se desvanecen las reglas, más bien se definen otras reglas nuevas. Así que el lenguaje peculiar en situaciones de disfunción resulta igualmente susceptible de análisis necesario. Pero además resulta imprescindible puesto que se convierte en indicador de la urgencia en disponer de datos lingüisticos en contexto si se ha de profundizar en los sistemas comunicativos propios de los trastornos, sobre los que precisamente se sustenta la decisión entre alternativas teóricas. De hecho, y como un factor más que refleja el valor intrínseco de las singularidades en trastornos como las afasias, se introducen dimensiones de variación que desplazan la disfunción más allá de la simple pérdida de reglas gramaticales típicas. Así, en una línea ya señalada por Caplan (1987: 338 y ss.), Lesser y Milroy (1993) subrayan el peso de ingredientes pragmáticos y sociolingüísticos que han de tomarse en cuenta para comprender y tratar las afasias. Los datos conversacionales en marcos naturales constatan destrezas comunicativas y señalan cauces de tratamiento. En su opinión:

The relatively recent development of a more liberal conception of the normal is highly relevant to language pathology, since clinicians with a unrealistic model of normal interaction are likely also to have difficulty in specifying accurately what is disordered, and may demand of their patients strange and unnatural skills which do not form part of the repertoire of normal conversationalists (...). The clinician must next decide whether normal or effective communication is the focus of therapy (Lesser y Milroy, 1993: 50). 
Asimismo, y en cuanto al habla peculiar en desarrollo, hay que subrayar el hito que marca el libro, que en su momento (1983) no tuvo el eco que debiera, de Peters sobre The units of language acquisition (reeditado de nuevo en 2002). Por revalorizar el lenguaje idiosincrático infantil como objeto de interés metodológico, el trabajo de Peters es pionero. No solo porque la autora diferencia entre: (a) unidades lingüísticas desde el prisma del niño, (b) unidades lingüísticas desde el prisma del adulto y (c) unidades desde el prisma de la Lingüística, sino también porque aborda cómo delimitar y definir categorias en el habla en proceso. Con sus palabras,

we must approach the study of language acquisition from the child's point of view, being aware of and trying to avoid any preconceptions derived from linguist's description of the adult language system. Only from observations that are interpreted on the basis of this principle can we justify inferences about the processes of language acquisition (Peters, 1983: 97).

Considera Peters que es necesario contemplar la Gestalt de la dinámica adquisitiva para encarar el análisis sin distorsionar lo genuino del habla infantil, por lo que duda de la conveniencia de usar conceptos como palabra y sintaxis en el desarrollo ${ }^{8}$. Parecen más adecuados conceptos menos comprometidos como unidad y construcciones. Las expresiones infantiles imitadas y creadas no se reconocen en su organización interna, sino que tienen valor por su cometido global. Así que lo interesante son los procesos que edifican y sostienen las emisiones. Señala Peters un primer proceso de "extracción" de piezas/unidades variables en longitud, y que da lugar a enunciados comunicativos (lo que Bateson llama praxon, Brown, chunk, y es holofrase para otros). Se trata en cualquier caso de una unidad o pieza que soporta un mensaje complejo. Para reconocer categorias propias en el habla infantil, la autora proporciona, entre otros, los siguientes criterios:

(a) ¿Es la expresión una cadena idiosincrática que el niño usa repetidamente y del mismo modo?

(b) ¿La construcción de la expresión no está relacionada con los patrones habituales del niño? (estereotipos y fórmulas que no se pueden tomar como prueba de que el niño dispone de esas reglas)

(c) ¿Tiene la expresión coherencia fónica (entonación, fluidez...)?

(d) ¿El uso de la expresión está sujeto a la situación en que se halle el niño?

(e) ¿Es la expresión una fórmula social?

Sobre estas bases, es posible identificar unidades, procesos y construcciones propios del habla infantil, que no son coincidentes con el habla

\footnotetext{
${ }^{8}$ Dice Peters con claridad rotunda que "To understand early language acquisition, one must understand the language units that children are actually working with. There is no reason to asume that these are necessarily the same as the units of adult language" (Peters, 1983: "Preface").
} 
adulta ni tampoco han de corresponder a las habitualmente establecidas en Lingüistica: "The first units of language acquired by children do not necessarily correspond to the minimal units (morphemes) of language described by conventional linguistics (Peters, 1983: 89).

En paralelo con la concepción detentada por Peters (1983) de valorar el habla infantil en su relevancia intrínseca, están las aproximaciones de expertos en lenguaje en desarrollo como, entre otros, Clark y MacWhinney ${ }^{9}$, quienes defienden el enfoque de procesos antes que establecer categorias. Se analizan así procesos "de sustitución", "de analogía", "de reducción" en la dinámica de desarrollo fónico y con objeto de hallar patrones y moldes sistemáticos que constaten las reglas propias de la dimensión fónica en periodo de desarrollo. Las aportaciones de Ingram (1981) y de Vihman (1996, $2010,2014)$ se han vuelto claves en esta ruta metodológica que facilita el estudio de las producciones fónicas en período infantil sin menoscabo de su dinamismo cambiante. También en la vertiente de construcciones se hace discurrir el análisis por cauces procesuales y mediante la consideración de procesos de predicación, procesos de combinatoria, y procesos de abstracción y concordancia, que corren a la par de la edificación paulatina de "gramáticas" por etapas. Las aportaciones de Brown (1973), Lieven (2010) y Tomasello (2003) son representativas de este modo de aproximarse al desarrollo gramatical respetando las singularidades de las estructuras emergentes en edad temprana. En conjunto, todos estos procedimientos se acomodan a estrategias de edificación gradual enmarcadas en la llamada teoria del andamiaje ya enunciada por Bruner (1975) sobre el concepto de zona de desarrollo próximo de Vigotsky (1934). Las técnicas de "corta y pega" que Tomasello revela en marcos de construcción que se van asentando son buena prueba del interés intrínseco de las tácticas gramaticales en edad infantil ${ }^{10}$. Secuencias como Mi papá tabaja en las vacas/ Fui a un caballo pequeñito poni evidencian tales tácticas en la construcción de estructuras que depositan el esquema argumental frecuente del predicado (trabajar en" / "ir a") y "pegan" segmentos que no se corresponden con los requisitos del hueco funcional.

El giro que se ha dado en la investigación sobre la adquisición del lenguaje, con el acento puesto en la relevancia propia de las peculiaridades del habla infantil, ha desplazado el foco de interés en cuanto a visiones teóricas basadas en tomar la lengua adulta conformada como espejo. Con palabras de Peters (1983: 5.3), "These contributions should provide a basis

\footnotetext{
${ }^{9}$ De Clark $(2003,2016)$ procede la afirmación de que «Los niños no nacen hablando. Aunque todos los niños hablan, lo importante es que no todos hablan igual". Por su parte, MacWhinney lidera y gestiona desde los años 90 la plataforma en la web que contiene el mayor número de repertorios de habla peculiar -sea lenguaje infantil, sea lenguaje disfuncional 3-4 -en el sitio en la red CHILDES-TalkBank (https://childes.talkbank.org/).

10 Tomasello (2003: 307 y ss.) considera las tácticas de filling in/ adding on como esenciales para el avance hacia las reglas sintácticas: se trata de ensayar ampliaciones de las estructuras básicas mediante procedimientos de inserción y secuenciación aun cuando los segmentos añadidos no respeten los requisitos distribucionales.
} 
for a more realistic modeling of language acquisition than approaches in which children are assumed to begin with precisely the lexical items of adult language".

Una consecuencia inmediata del cambio de mirada hacia el valor intrínseco del habla infantil es el desplazamiento de las apreciaciones sobre cómo se expresan los niños. Desaparece así el calificativo de "error" aplicado a las producciones verbales en las primeras etapas, y se disipan las inútiles tareas de corrección en estadios procesuales privativos ${ }^{11}$. Las singularidades propias del lenguaje infantil se contemplan como lo que son: rasgos que deben describirse por su relevancia en el sistema del que forman parte y que no se corresponden con la gramática de los adultos. En un trabajo reciente, Blume y Lust señalan que "Children must never feel they are making errors because they are not 3-4 they are demonstrating their natural language and the grammar for this language" (2017:76). De ahi que, si bien la etiqueta de "error" es habitual, lo conveniente es sustituirla por otros rótulos que se ajusten a la dinámica verbal de la adquisición. En opinión de las autoras:

Although the term error is frequently used, a more suitable term is deformation or reformation because these deformations are viewed as providing essential information on the subject's theory of grammar for the language (Blume y Lust 2017: 196, note 4).

En sentido similar se pronunciaban Lesser y Milroy (1993: 50) respecto de "errores" como repeticiones, expresiones incompletas, vacilaciones, en afasias, que de ningún modo pueden tildarse como tales a no ser que se interprete que en la conversación el molde es el modelo de lengua escrita para componer textos.

En la esfera del habla peculiar por disfunción, la rotación hacia la importancia de las producciones en marcos comunicativos contextualizados ha traído consigo el desplazamiento de los modos de evaluación. Los sistemas basados en cuestionarios con ítems que prevén respuestas cerradas de lengua típica se han ido sustituyendo por plantillas que requieren el depósito de datos ecológicos provenientes de producciones naturales. De las evaluaciones categóricas con expectativas de evaluar por "lo-que-le-falta" se ha pasado a las valoraciones graduales con el foco en cómo se comunica por "lo- que-tiene" a su disposición. Estimar los recursos comunicativos quiere decir que no se los considera errores, sino que se les atribuye significado y relevancia tanto para comprender las bases en que se asienta el potencial verbal del paciente, como para diseñar vías de terapia con arreglo a las tácticas comunicativas que ya posee ${ }^{12}$. Entre otros, son

\footnotetext{
${ }^{11}$ El propio Gili Gaya en su discurso ante la Academia aludia a las condiciones que se requieren en un maestro para cumplir su papel de guía: "El maestro que pretenda dirigir la actividad expresiva de los niños debe aprender primero a conocerla y respetarla" (Gili Gaya, 1961: 31).

12 El soberbio y monumental trabajo de Paul (1995) en su Language Disorders from Infance Through Adolescence. Assessment and Intervention contiene las lineas maestras de lo que debe
} 
excelentes instrumentos de evaluación basados en muestras verbales naturales The Clinical Assessment of Language Comprehension de Miller y Paul (1995), los que se ajustan a prácticas comunicativas como el CAPPA (Conversation Analysis Profile for People with Aphasia, del grupo de Lesser (cf. Whitworth et al., 1997), o, en general, las estimaciones que requieren perfiles evolutivos y que son comunes entre los grupos británicos en la estela de Crystal, como el LARPS (Language Assessment, Remediation and Screening Procedure, cf. Crystal et al., 1976), o el PACS (Phonological Assessment of Child Speech, cf. Grunwell, 1985).

\subsection{ENFOQUES TEÓRICOS EN LINGÜÍSTICA Y RELEVANCIA DEL HABLA PECULIAR}

El valor propio atribuido al lenguaje singular se hace patente en la lingüistica actual en todos los planteamientos ligados a inventarios de habla infantil (Blume y Lust, 2017), y que sin duda tienen su antecedente en la investigación conducida por Slobin entre 1985 y 1997, y que ha fructificado en los cinco volúmenes de The Crosslinguistic Study of Language Acquisition, si bien la disponibilidad de datos que hoy se oferta en los corpus de producciones infantiles revela con garantías la valorización del habla peculiar en estos últimos años.

La repercusión de los avances en Lingüística ha incidido de manera cada vez más decisiva en la consideración privativa del habla peculiar, pero en mayor medida ha percutido en el diseño de rutas metodológicas para abordarla y describirla. El peso de la pragmática ha sido capital. En la actualidad el grueso de los estudios sobre habla disfuncional o sobre lenguaje infantil comulgan con el denominado prisma pragmático que es el que facilita contemplar las producciones en el marco de su eficacia comunicativa. Si las emisiones consiguen efecto interactivo, hay que estudiarlas, y para ello hay que adoptar una perspectiva comunicativa transversal antes que ópticas parciales sobre dimensiones fónicas o gramaticales. Smith y Leinonen (1992: 143) hablan de revolución pragmática al constatar que en clínica no es posible divorciar el lenguaje de la comunicación, y señalan con claridad meridiana que

the study of 'pragmatics' is not a level of analysis (...). Rather, pragmatics constitutes a study which draws upon the various levels of linguistics for its data; a study which explores how the various levels of language are used for communicative ends (Smith y Leinonen, 1992: 41) ${ }^{13}$.

\footnotetext{
ser una aproximación basada en lo que el paciente tiene a su disposición y cómo recuperar si se explotan sus recursos.

${ }^{13}$ De hecho, hay dislalias que son solo disfunciones articulatorias mientras otras tienen origen o incidencia simbólica no solo limitada al significado léxico, sino con repercusión en gramática (sucede por ejemplo con el sonido [-s] en posición final en español, con trascendencia gramatical en concordancias de número y persona). La consideración comunicativa y transversal de los niveles de la lengua resulta clave; con palabras de Smith y Leinonen (1992: 145): "The interaction of linguistic levels gains particular significance in the assessment of linguistic skills and consequent diagnoses of problems".
} 
Además de la vitalidad por el progreso mismo de la lingüistica actual ya orientada hacia el habla (la parole/ the performance), el lenguaje peculiar se ha insuflado relevancia descriptiva gracias a planteamientos metodológicos que posibilitan su análisis. Entre esos enfoques que en la lingüística más reciente se acogen a las novedades del habla disfuncional y del habla en desarrollo, está la llamada lingüistica de construcciones (Goldberg 1995, Hoffman \& Trousdale 2013). Basada en el lema enunciado por Givón «El discurso de hoy es la gramática del mañana", y que se ajusta a técnicas de indagación bottom-up ${ }^{14}$, la lingüistica de construcciones se centra en el estudio de los usos verbales y de las acciones comunicativas reales para describir en ellos los niveles de eficacia y éxito interactivo. Puede decirse que son los marcos pragmáticos los idóneos para abordar la consideración y el análisis de las producciones idiosincráticas de los sujetos. Hay que estudiar las funciones en el discurso porque sobre ellas se sostienen las reglas formales que se van elaborando.

Las quasi-gramáticas, proto-gramáticas o gramáticas especiales que se sostienen en el habla peculiar están edificadas sobre el discurso. Son gramáticas emergentes que no están definidas previamente, sino que se construyen de modo gradual a través de los usos. Con palabras de Hopper (1998: 156):

Grammar, in this view, is not the source of understanding and communication but a by-product of it. Grammar is, in other words, epiphenomenal (...) its structure is always deferred, always in a process bur never arriving, and therefore emergent ${ }^{15}$.

En esa dinámica de edificación de construcciones que sostienen mensajes comunicativos, pueden observarse estrategias como las que conducen a las denominadas por Tomasello (1992) verb island, o las técnicas de corta y pega (cutting and pasting, Tomasello 2003), o los procesos graduales que se plasman en cada componente de la lengua. Que las

${ }^{14}$ Kemmer y Barlow (1999: ix-x) definen esta táctica en los términos siguientes:

The bottom up property adds that the specific and idiosyncratic elements of the system are privileged over the general in the acquisition and operation of the system: the general arises out of the specific, and the specific is what is most directly taken from experience (destacado nuestro).

15 Obviamente, estas gramáticas no son las esperadas en el lenguaje típico, tampoco las gramáticas descriptivas de la lingüística. No sólo Peters (1983) ha puesto el acento en la necesidad de evitar el reduccionismo entre lo que es ontologia (habla peculiar y habla típica) y lo que es metodología (gramática descriptiva del lingüista), sino que también Slobin (2001) alude, no sin cierta ironía, a la necesidad del rigor y de la precisión con apreciaciones como las siguientes:

The modules that are postulated often have names that evoke suspicion: they are names of our own academic fields (linguistics, mathematics, physics, biology), or subfields (clossed-class morphemes, grammaticizable notions). Could God or evolution have anticipated the academic and intelectual organization of late twentieth-century America? (Slobin, 2001: 442-443). 
emisiones con un único predicado tengan relevancia descriptiva (toma/ tae/ mujer come), que las estructuras no limiten formalmente sus requisitos, sino que admitan técnicas de "corta y pega" (mirar en un caballo/ trabajar en las vacas), o que se describan procesos fónicos (de reducción en [apáto]; de lateralización en [atlás], de frontalización y reducción en [síne bánko]), y procesos gramaticales (de predicación en toma/ tae; de combinatoria en apato nena/ senta silla; o de concordancia en el noche/ lefante buenos) hacen patente la importancia del lenguaje peculiar habida cuenta del interés descriptivo de sus características. No obstante, no debiera reducirse la aportación de la lingüística de construcciones a únicamente el cauce metodológico que proporciona para abordar el análisis de las producciones peculiares, sino que debe extenderse, por su trascendencia, al peso representacional de su modelo que es reflejo del habla en evolución y en cambio. Precisamente, el lenguaje peculiar en desarrollo y en dinámicas de disfunción exige aproximaciones que de ningún modo pueden ser estáticas ni tampoco instantáneas. Sea lenguaje infantil, sea lenguaje disfuncional, se hace imprescindible un seguimiento evolutivo que permita vislumbrar los cambios procesuales en marcha, y en la lingüistica de construcciones los modelos de protogramáticas emergentes convierten en natural lo que únicamente parecía una condición extravagante en casos anómalos.

\section{Conclusiones. A modo de corolario}

- El habla peculiar tiene, en la actualidad, su lugar en lingüística, tal y como entonces deseaba en 1956 Jakobson: "La Linguistique s'intéresse au langage sous tous les aspects - au langage en acte, au langage en évolution, au langage à l'état naissant, au langage en dissolution" (1956: 43). Y ya no solo desde la institucionalización de la esfera de Terapéutica del habla en la primera mitad del s. XX, sino además por la presencia indiscutible del lenguaje peculiar valorado en sus vertientes simbólicocognitivas en la lingüística de hoy.

- Son los grandes repertorios de datos de habla especial compilados (tanto procedentes de dinámicas de desarrollo verbal infantil como derivados de situaciones disfuncionales) los que facilitan el acceso a las características genuinas de las producciones. Los inventarios de habla peculiar son manantiales que permiten constatar el valor de tales propiedades. La Lingüística de corpus ha sido clave en su dotación de prismas y medios para institucionalizar el interés del lenguaje singular. La documentación en fuentes de datos visibiliza su importancia y la previsible riqueza de las descripciones.

- La perspectiva comunicativa (el denominado prisma pragmático, Smith y Leinonen, 1992) ha proporcionado fundamento tanto a la "calidad" de las producciones ya que disfrutan de eficacia comunicativa, como a los cauces de registro del habla peculiar que interesan por sus caracteristicas privativas. 
- En la lingüística más reciente hay enfoques teórico-metodológicos (como la llamada lingüística de los usos o lingüistica de construcciones) que canalizan los instrumentos descriptivos necesarios para analizar el habla peculiar. La orientación bottom-up promueve el relieve de lo singular: "the specific and idiosyncratic elements of the system are privileged over the general in the acquisition and operation of the system: the general arises out of the specific, and the specific is what is most directly taken from experience" (Kemmer y Barlow, 1999: ix-x). El análisis de procesos y de construcciones favorece la representación dinámica de las cadencias evolutivas esenciales en la comunicación en general y en el habla idiosincrática en particular.

- La investigación del habla peculiar en sus aspectos privativos y en sus propiedades intrínsecas, y sin renunciar a la diversidad consecuencia de factores interlingüísticos y contextuales, ha actuado como revulsivo tanto para destacar procesos y estrategias en la teoría sobre la adquisición de la lengua, como para redefinir generalizaciones sobre las disfunciones formuladas en máximos (como ha sido el caso del agramatismo, o la reorientación de las evaluaciones a base de cuestionarios referenciados en categorías de lengua esperable). Las consideraciones de Bates, Devescovi y Wulfeck (2001) sobre la urgencia de una psicolingüística de planta comparativa entre lenguas resultan incontestables:

Overall processability is the product of cost-benefit tradeoffs, a constraint satisfaction problem that must be solved across multiple dimensions of the language system. As a result, we may obtain powerful differences between languages in the relative difficulty of specific linguistic structures, with differential effects on performance by children, aphasic patients, and healthy normal adults (Bates et al., 2001: 374).

- Uno de los efectos capitales del reconocimiento descriptivo del habla peculiar es la incidencia en los sistemas evaluativos para estimar su estado y su evolución. De los planteamientos en términos de máximos formales que evalúan por "lo-que-le-falta" se ha pasado a estimaciones graduales con interés en cómo se comunica por "lo-que-tiene" a su disposición. Valorar los recursos comunicativos quiere decir que no se los considera "errores", sino que se admite su relevancia tanto para comprender las bases en que se asienta el potencial verbal del paciente, como para diseñar rutas de terapia con arreglo a las tácticas comunicativas que ya posee.

\section{REFERENCIAS}

$\begin{array}{ccc}\text { ALARCOS LLORACH, } & \text { E. } & \text { Tratado del lenguaje, Buenos } \\ \text { (1968) : "L'acquisition } & \text { du } & \text { Aires: Nueva Visión, 1976, pp. } \\ \text { langage par l'enfant", Martinet, } & 9-42 . \\ \text { A. (ed.): Le langage, Paris: } & \text { BARNILS, P. (1930): Defectes del } \\ \text { Gallimard, pp. 325-365. } & \text { parlar, Barcelona: Llibreria } \\ \text { Traducción de H. Acevedo, } & \text { Catalònia. }\end{array}$


BATES, E.; DEVESCOVI, A. \& WULFECK, B. (2001): "Psycholinguistics: A CrossLanguage Perspective", Annual Review of Psychology, 52, pp. 369-396.

BLUME, M. \& LUST, B. (2017): Research Methods in Language Acquisition. Principles, Procedures and Practices, Berlin: De Gruyter Mouton.

BROWN, R. (1973): A first language: the early stages, Cambridge: The Harvard University Press.

BRUNER, J. (1975): "From communication to language: A psychological perspective", Cognition, 3, pp. 255-287.

CAPLAN, D. (1987): Neurolinguistics and Linguistic Aphasiology, Cambridge: Cambridge University Press. Traducción al español de L. Eguren, Introducción a la Neurolingüistica y al estudio de los trastornos del lenguaje, Madrid: Visor, 1992.

CLARK, E. V. (2003): First Language Acquisition, Cambridge, Cambridge University Press.

CLARK, E. V. (2016): Language in Children, London: Routledge.

CRYSTAL, D. (1980): Introduction to Language Pathology, London: E. Arnold. Traducción de M. L. Sánchez Bernardos, Patología del lenguaje, Madrid: Cátedra, 1983.

CRYSTAL, D.; FLETCHER, P. \& GARMAN, M. (1976): The Grammatical Analysis of Language Disability, London: Edward Arnold.

ELDRIDGE, M. (1968): A history of the treatment of speech disorders, Edinburgh: Livingstone.

GILI GAYA, S. (1961): Imitación y creación en el habla infantil, Madrid: Real Academia Española
GIVÓN, T. (2002): “The visual information-processing system as an evolutionary precursor of human language", Givón, T. \& Malle, B. (eds.): The Evolution of Language out of Pre-language, Amsterdam: John Benjamins, pp. 3-50.

GOLDBERG, A. (1995): Constructions. A construction grammar approach to argument structure, Chicago: University of Chicago Press.

GOLDSTEIN, K. (1948): Language and Language Disturbances, New York: Grune and Stratton.

GRODZINSKY, Y. (1984): "The syntactic characterization of agrammatism", Cognition, 16 (2), pp. 99-120.

GRUNWELL, P. (1985): Phonological Assessment of Child Speech (PACS), Windsor: NFER-Nelson.

HOFFMAN, T. \& TROUSDALE, G. (eds.) (2013): The Oxford Handbook of Construction Grammar, Oxford: Oxford University Press.

HOPPER, P. J. (1998): "Emergent Grammar", Tomasello, M. (ed.): The New Psychology of Language. Cognitive and Functional Approaches to Language Structure, N. Jersey: Lawrence Erlbaum, pp. 155175.

HOWARD, D. \& HATFIELD, F. (1987): Aphasia Therapy. Historical and Contemporary Issues, Hove: Lawrence Erlbaum.

INGRAM, D. (1981): Procedures for the Phonological Analysis of Children's Language, Baltimore: University Park Press.

JACKSON, J. H. (1879): "Reprints of H. Jackson's Papers on affections of speech, Brain, 38 (1-2), 1915, pp. 1-190.

JAKOBSON, R.

Kindersprache, Aphasie und 
Allgemeine Lautgesetze, Uppsala, Universitets Aarsskrift. Traducción al español de E. Benítez (sobre la versión francesa), Lenguaje infantily afasia, Madrid: Ayuso, 1974.

JAKOBSON, R. (1956): "Deux aspects $\mathrm{du}$ langage et deux types d'aphasies", Essais de Linguistique Générale. Traduit par N. Ruwet de l'original, Fundamentals of Language, The Hague, Paris: Éditions de Minuit, 1963, pp. 43-6.

KEMMER, S. \& BARLOW, M. (1999): "Introduction: A Usage-Based Conception of Language", Barlow, M. \& Kemmer, S. (eds.): Usage based models of language, Stanford: Center for the Study of Language and Information, pp. vii-xxviii.

LESSER, R. \& MILROY, L. (1993): Linguistics and Aphasia: Psycholinguistic and pragmatic aspects of intervention, London: Longman.

LIEVEN, E. (2010): “Language development in a crosslinguistic context", Kail, M. \& Hickmann, M. (eds.): Language Acquisition across Linguistic and Cognitive Systems, Amsterdam: John Benjamins, pp. 91-108.

LURIA, A. (1975): "Neuropschology: its sources, principles and prospects", Worden, F., Swazey, J. \& Adelman, G. (eds.): The Neurosciences: Paths of Discovery, Cambridge: The MIT Press, pp. 335-361.

MENN, L. \& OBLER, L. (eds.) (1990): Agrammatic Aphasia. A CrossLanguage Narrative Sourcebook, 3 vols., Amsterdam: John Benjamins.

MILLER, J. \& PAUL, R. (1995): The Clinical Assessment of Language Comprehension, Baltimore: Paul H. Brookes.
MORLEY, M. (1957): Disorders of Speech in Childhood, London: Livingstone.

PAUL, R. (2007 $\left.{ }^{3}\right)$ : Language Disorders from Infancy through Adolescence, St. Louis: Mosby Elsevier.

PETERS, A. (1983): The Units of Language Acquisition, Cambridge: Cambridge University Press (Hawai University Press, 2002).

SLOBIN, D. (1985-1997): The crosslinguistic study of language acquisition, 1-5, Mahwah, New Jersey: Lawrence Erlbaum.

SLOBIN, D. (2001): "Form-function relations: how do children find out what they are?", Bowerman, M. \& Levinson, S. C. (eds.): Language acquisition and conceptual development, Cambridge: University Press, pp. 406-449.

SMITH, B. \& LEINONEN, E. (1992): Clinical Pragmatics. Unravelling the complexities of communicative failure, London: Chapman \& Hall.

TEMPLIN, M. (1957): Certain Language Skills in Children, Minneapolis: University of Minnesota Press.

TOMASELLO, M. (1992): First Verbs. A Case Study in Early Grammatical Development, Cambridge: Cambridge University Press.

TOMASELLO, M. (2003): Constructing a Language: A Usage-based Theory of Language Acquisition, Cambridge: MA: Harvard University Press.

VIHMAN, M. (1996): Phonological Development: The origins of language in the child, Oxford: Wiley-Blackwell.

VIHMAN, M. (2010): "Phonological templates in early words: A cross-linguistic study", Fougeron, C., Kühnert, B., 
D’imperio, M. \& Vallée, N. (eds.): Laboratory Phonology 10, New York: De Gruyter, pp. 109133.

VIHMAN, M. (2014): Phonological Development: The first two years, Malden: WileyBlackwell.

VIHMAN, M. \& CROFT, W. (2007), "Phonological development: Toward a 'radical' templatic phonology", Linguistics, 45, pp. 683-725.

VYGOTSKI, L. (1934): Myshlenie $i$ rech, Mockba. Traducción de P. Tosaus Abadía (sobre la edición inglesa a cargo de A. Kozulin, Thought and language, Cambridge: The MIT Press, 1986), Pensamiento y lenguaje, Barcelona: Paidós, 1995.

WHITWORTH, A.; PERKINS, L. \& LESSER, R. (1997): CAPPA (Conversation Analysis Profile for People with Aphasia), London: Whurr Publishers.

ZANGWILL, O. (1947): "Psychology, speech therapy and rehabilitation", Speech, 11, pp. 4-8. 